\title{
A systemic analysis of typhoon risk across China
}

\author{
Xinliang Xu • Daowei Sun • Tengjiao Guo
}

Received: 5 August 2014/Accepted: 3 January 2015/Published online: 11 January 2015

(C) Springer Science+Business Media Dordrecht 2015

\begin{abstract}
China is one of the countries that have been continuously experiencing negative impacts by typhoons. Typhoons cause severe damage annually, not only to the regional economy, but they are also responsible for frequent human casualties. This research comprehensively assesses the impact of typhoons across China by using the geographical information system based on time series data between 1980 and 2012. Results indicate that typhoons affected $61 \%$ of the total landmass of China during this timeframe with a total affected area of approximately 5.91 million $\mathrm{km}^{2}$. In addition, some coastal regions of south-east China experienced nearly 300 typhoon landfalls. The key entities of this study that are used to assess typhoon risk across China include population, gross domestic production, road networks and land use. As a result, the vulnerabilities of each entity in the typhoon-affected region of China were calculated before leading to the regional typhoon risk indexes. Based on the range of typhoon risk indexes, the typhoonaffected areas in China were zoned into four regions with different levels of typhoon risk. This research will facilitate decision-making by policy makers on the mitigation of typhoon risks.
\end{abstract}

Keywords Typhoon hazards - Disaster frequency index · Vulnerability - Typhoon risk

\footnotetext{
$\mathrm{X} . \mathrm{Xu}$

State Key Laboratory of Resources and Environmental Information System, Institute of Geographical Sciences and Natural Resources Research, Chinese Academy of Sciences, 11 Datun Road Chaoyang District, Beijing 100101, China

e-mail: xuxl@1reis.ac.cn

D. Sun $(\bowtie)$

School of Marketing and Management, The University of Adelaide, 10 Pulteney Street, Adelaide, SA 5005, Australia

e-mail: daowei.sun@adelaide.edu.au

T. Guo

School of Population, Resources and Environment, Shandong Normal University, 88 East Weihua Road, Jinan 250014, Shandong, China

e-mail: guotengjiao@126.com
} 


\section{Introduction}

A typhoon is a mature tropical cyclone which causes high-speed wind, storm tide and heavy rainfall. Typhoons are considered to be extremely lethal geophysical hazards, which originate over tropical oceans and are driven principally by heat transfer from the ocean (Emanuel 2003). However, kind of natural hazard extends far beyond the tropical regions due to its movement impact (Jones et al. 2003). Due to the devastating impact of typhoons, the research on typhoons has long been a hot topic for researchers from various disciplines (Chu et al. 2012; Hashimura and Takikawa 2012; Meng et al. 2002; Xu et al. 2014; Zhang et al. 2013; Zhou et al. 2012). It is believed that typhoons would become more severe in the light of current of climate change forecasts (Emanuel 2005; Wang et al. 2007).

China is one of the countries that have been experiencing frequent typhoon landfalls each year, especially in the south-east coastline regions (Zhang et al. 2011). Unfortunately, these coastlines accommodate the most developed economy within China (Naughton 2007). For this reason, the damage caused by typhoons has been accumulating due to the rapid urbanisation in these regions. This includes heavy losses of property, severely damaged infrastructure and frequent human casualties after each typhoon landfall (Liu et al. 2009; Niu et al. 2011; Wang et al. 2007; Xinhua News 2014a, b).

From a policy maker's perspective, there is a need to understand the entity vulnerability to typhoons and the regional risk for effective natural hazard prevention (UNDP/BCPR 2004). Unfortunately, most of research on typhoons in China is focussed on small regions, generally within one or two cities or a couple of provinces (Liu et al. 2012; Wang et al. 2013; Zhang et al. 2011). However, this research uses time-series data over a period of 32 years to access the impact of typhoons across all of China. In contrast, research has proven that a typhoon's ripple impact extends far beyond the coastline (Fumin et al. 2002). Therefore, a holistic study of typhoon risk across China is urgently needed. Assessing and predicting the risk of typhoons are the major interest of researchers. Extensive research on typhoon risks can be found using approaches involving statistical data analysis, simulation modelling and the use of geographical information system (GIS; Chia and Ropelewski 2002; Matsuura et al. 2003; Peduzzi et al. 2009; Wu et al. 2006). In terms of methods of research into natural hazard risks, it is highly encouraged and valued by the United Nations to map the vulnerability of the natural hazards affected entities as well as the hazard risk using GIS mapping techniques (ISDR 2004). However, the work has not yet been conducted in China.

In order to address the disparity between the extensive typhoon impacts and localised typhoon research within China, this research presents a systematic assessment of typhoon risks across China using the GIS method. Priorities were placed on calculating the vulnerabilities of selected entities as well as the regional typhoon risk across all the regions that have been affected by typhoons.

\section{Methods and materials}

\subsection{Research method}

The conventional notion of risk is expressed in Eq. (1) which was defined by International Strategy for Disaster Reduction (ISDR 2004). 


$$
\text { Risk }=\text { Hazards } \times \text { Vulnerability }
$$

Where Risk is the probability of harmful consequences or expected losses resulting from interactions between natural or human-induced hazards and vulnerable conditions; Hazards are potentially damaging events, phenomena or human activities; and Vulnerability is the degree of susceptibility of the elements exposed to hazards.

This is to say that the risk of a natural hazard will depend on the characteristics, probability and intensity of the hazard, as well as the susceptibility of the exposed elements based on physical, social, economic and environmental conditions. In practice, the selection of parameters to assess the risk of a given natural hazard to entities is generally situation based (Feng and Luo 2009; Liu et al. 2013; Tillman et al. 2010; Zhang et al. 2014). The selection of parameters normally needs to consider factors such as the nature of the hazard; the scale of the entity that is affected by the hazard; and the availability of the data set of different natural social and economic parameters. For instance, the factor of exposure may be taken into consideration separately, or it can also be reflected in the process of calculation of vulnerability (Davidson and Lambert 2001; ISDR 2004).

In this research to assess the risk of typhoons, we follow the common practice of many researchers in calculating the risk index using the Eqs. 2-4 (Chen and Zhong 2010; Du and Lin 2012; Ni and Nan 2014; Tsai and Chen 2011).

$$
\begin{gathered}
R=(T / n) \times C \\
T=\sum N \\
C=\sum W_{i} \times D_{i}
\end{gathered}
$$

where $R$ is the disaster risk index; $T$ is the hazard index (times); $n$ is length of studied period (years); $C$ is the vulnerability of entities; $N$ is the number of hazards happened in a given region during a period (times/year); $W_{i}$ is the weight of the vulnerability of the entity $i$ to typhoons; and $D_{i}$ is unit-less scores of entity $i$ using linear scaling function.

\subsection{Parameters and data sources}

\subsubsection{Parameter system and weights determination}

The process of selecting entities across China for assessment of typhoon risk and the determination of their respective weights was based on a series of organised workshops. Participants invited for workshops were from a wide range of academic backgrounds and different sectors in order to minimise possible bias towards the determination of parameters and weights. The academic backgrounds of workshop participants included physical geography, meteorology, agriculture, natural hazard prevention, social economics, environmental sciences, environmental management, civil engineering and hydrology. The social roles of these workshop participants included academics from universities and research institutions, as well as related governmental officers. Public consultations in the coastal regions of the south-eastern China also contributed to the determination of the weights for each of the selected parameters. Based on the significance and accessibility of entities exposed to typhoons, four categories of entities at first level were selected which were the population, gross domestic production (GDP), road network and land use. Both 
the road network and the land use were further developed into their second-level parameters taking into consideration different types of roads or land uses showing different levels of vulnerability. Road networks were further categorised into highways, national roads, provincial roads and county roads/township roads, which formed the second level of parameters. Land uses were also further developed into six second-level parameters that included residential land, farmland, forestry, grassland, water bodies and other land. All the entities and their parameters exposed to typhoons are summarised in Table 1. The calculation of weights for each parameter was based on expert opinion that was then processed using the analytic hierarchy process (Dong et al. 2008; Saaty 2008). Weights for each of the calculated parameters are also listed in Table 1.

\subsubsection{Data sources}

Data on typhoon characteristics were sourced from the National Institute of Informatics in Japan, which cover all the typhoon landfalls across China between 1980 and 2012. The affected range of each typhoon was calculated on the basis of typhoon radius, using the Beaufort scale for wind over seven (wind speed over $14 \mathrm{~m} / \mathrm{s}$ ). The Buffer function, a spatial analysis function, within the ArcGIS platform was used to define the range of each typhoon on a map. By overlapping the ranges of all the typhoons that happened between 1980 and 2012, a map was obtained which shows typhoon frequency in any given region across China.

Data relating to land use were sourced from the Data Centre for Resources and Environmental Sciences Chinese Academy of Sciences (RESDC), which is a 2010 land-use database, with a mapping scale of 1:1,000,000. The land-use data were classified into 25 categories which were subsequently grouped into six types: cropland, woodland, grassland, water body, built-up area and unused land. Detailed information about this land-use database can be found in previous reports (Liu et al. 2014, 2010). The data of land use at county level across China were calculated. On the other hand, the data of road network, population and GDP were sourced from national statistics of year 2010 (State Statistic

Table 1 The built parameter system with weight matrix

\begin{tabular}{lllll}
\hline First-level parameters & Weights & Second-level parameters & Weights & Total weights \\
\hline Population & 0.47 & & & 0.4700 \\
GDP & 0.28 & & & 0.2800 \\
Road network & 0.16 & Highways & 0.47 & 0.0752 \\
& & State roads & 0.28 & 0.0448 \\
& & Provincial roads & 0.16 & 0.0256 \\
& \multirow{2}{*}{0.09} & County/township roads & 0.09 & 0.0144 \\
Land use & Urban and residential land & 0.38 & 0.0342 \\
& & Farm land & 0.25 & 0.0225 \\
& & Forestry & 0.16 & 0.0144 \\
& & Grassland & 0.10 & 0.0090 \\
& & Water bodies & 0.07 & 0.0063 \\
& & Other land & 0.04 & 0.0036 \\
\hline
\end{tabular}


Table 2 Typhoon hazard index and land areas affected by typhoons between 1980 and 2012

\begin{tabular}{lll}
\hline $\begin{array}{l}\text { Typhoon } \\
\text { hazard index }\end{array}$ & $\begin{array}{l}\text { Area affected by } \\
\left.\text { typhoons (million } \mathrm{km}^{2}\right)\end{array}$ & Provinces affected by typhoons \\
\hline $1-30$ & 3.76 & $\begin{array}{l}\text { Yunnan, Guizhou, Chongqing, Sichuan, Wuhan, Qinghai, } \\
\text { Gansu, Ningxia, Shanxi, Henan, Shanaxi, Shandong, Beijing, } \\
\text { Tianjin, Hebei, Inner Mongolia, Jilin, Liaoning, Heilongjiang } \\
\text { Guangxi, Yunnan, Tibet, Guizhou, Hubei, Hunan, Anhui, } \\
\text { Henan, Jiangsu, Shandong, Jilin, Liaoning, Heilongjiang }\end{array}$ \\
$30-80$ & 1.22 & $\begin{array}{l}\text { Jiangsu, Anhui, Shanghai, Zhejiang, Fujian, Guangdong, } \\
\text { Guangxi, Hunan, Jiangxi, Hainan }\end{array}$ \\
$150-200$ & 0.06 & $\begin{array}{l}\text { Taiwan, Fujian, Guangdong, Hainan } \\
\text { Taiwan }\end{array}$ \\
\hline $200-296$ & 0.02 &
\end{tabular}

Bureau 2011), which were also calculated at the county level. The calculation of typhoon risk indexes involved the calculation of vulnerabilities of selected entities of population, GDP, road network and land use, respectively. By using the spatial analysis function within the ArcGIS platform, the overall vulnerability of entities was calculated by overlapping the vulnerabilities of these four types of entities following the Eq. (4).

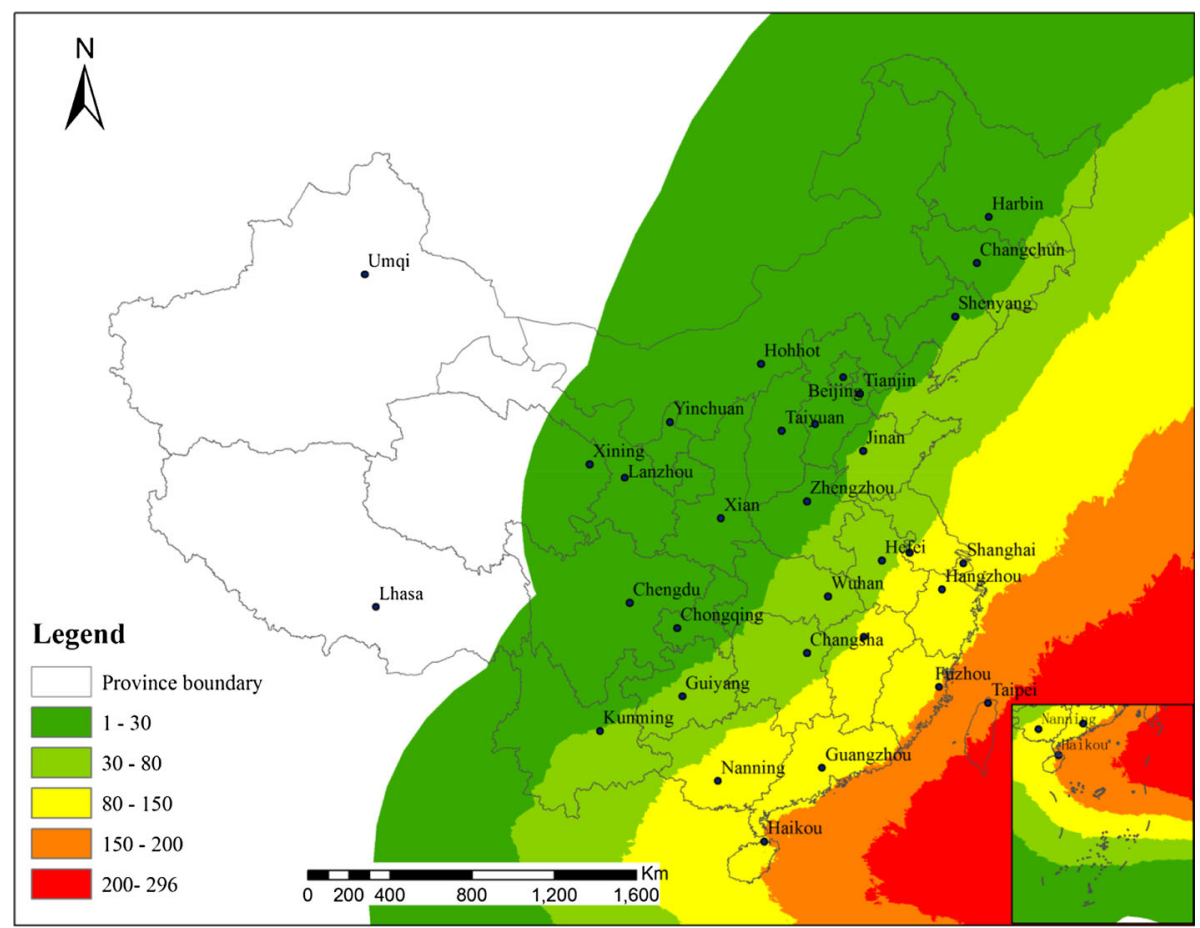

Fig. 1 Typhoon hazard index $(T)$ across typhoon-affected regions in China. Based on typhoon data during the period 1980-2012 


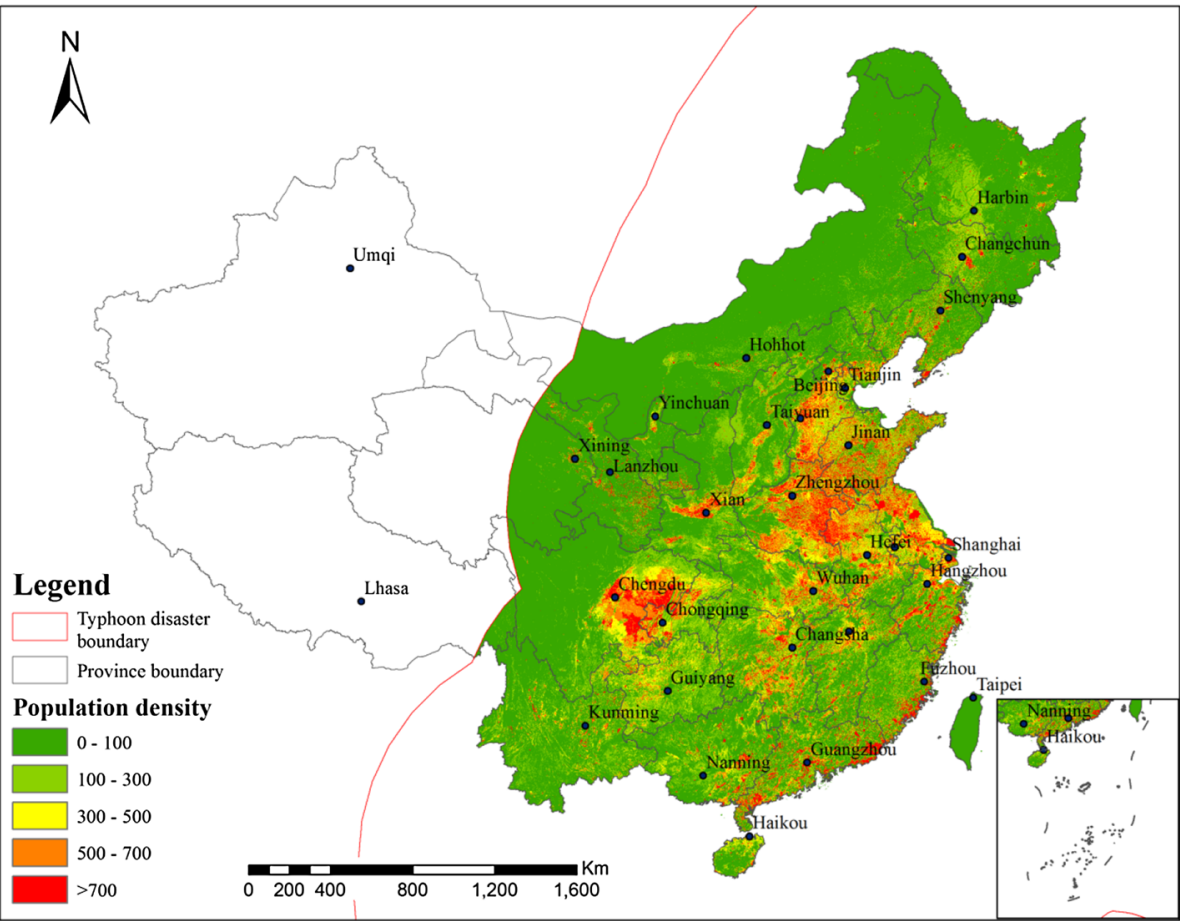

Fig. 2 The distribution of population density in typhoon-affected regions

\section{Results and analysis}

\subsection{The typhoon hazards}

By closely examining all the typhoon landfalls in China during years 1980-2012, three kinds of patterns of the typhoon tracks were identified. The first pattern is when a typhoon moves from the sea to the east of the Philippines in a westerly direction it pasts the South China Sea and makes landfall in either Hainan province or Guangdong province. There were approximately $19 \%$ of typhoons that followed this track. The second track formed by typhoons move from the sea to the east of the Philippines towards the north-west and make landfall in either Taiwan or Fujian or Zhejiang province. Approximately $27 \%$ of typhoons followed this track and mainly occurred between July and September each year. The third pattern is that typhoon tracks show a projectile shape which means after typhoons are formed from the ocean to the east of the Philippines, they firstly move towards north-west, then move northwards and finally towards north-east. On the average, there were eight typhoon landfalls in China annually.

In terms of the area affected by typhoons between the years 1980 and 2012, the total area was 5.91 million $\mathrm{km}^{2}$, which means $61 \%$ of the land area of China was affected by typhoons. The most severely affected areas, in which the typhoon hazard index was larger than 150, covered 0.08 million $\mathrm{km}^{2}$, which included Taiwan, Fujian, Guangdong and Hainan provinces (Table 2). On the other hand, the areas which had a typhoon hazard index between 80 and 150 covered 0.85 million $\mathrm{km}^{2}$ and included 10 provinces. 


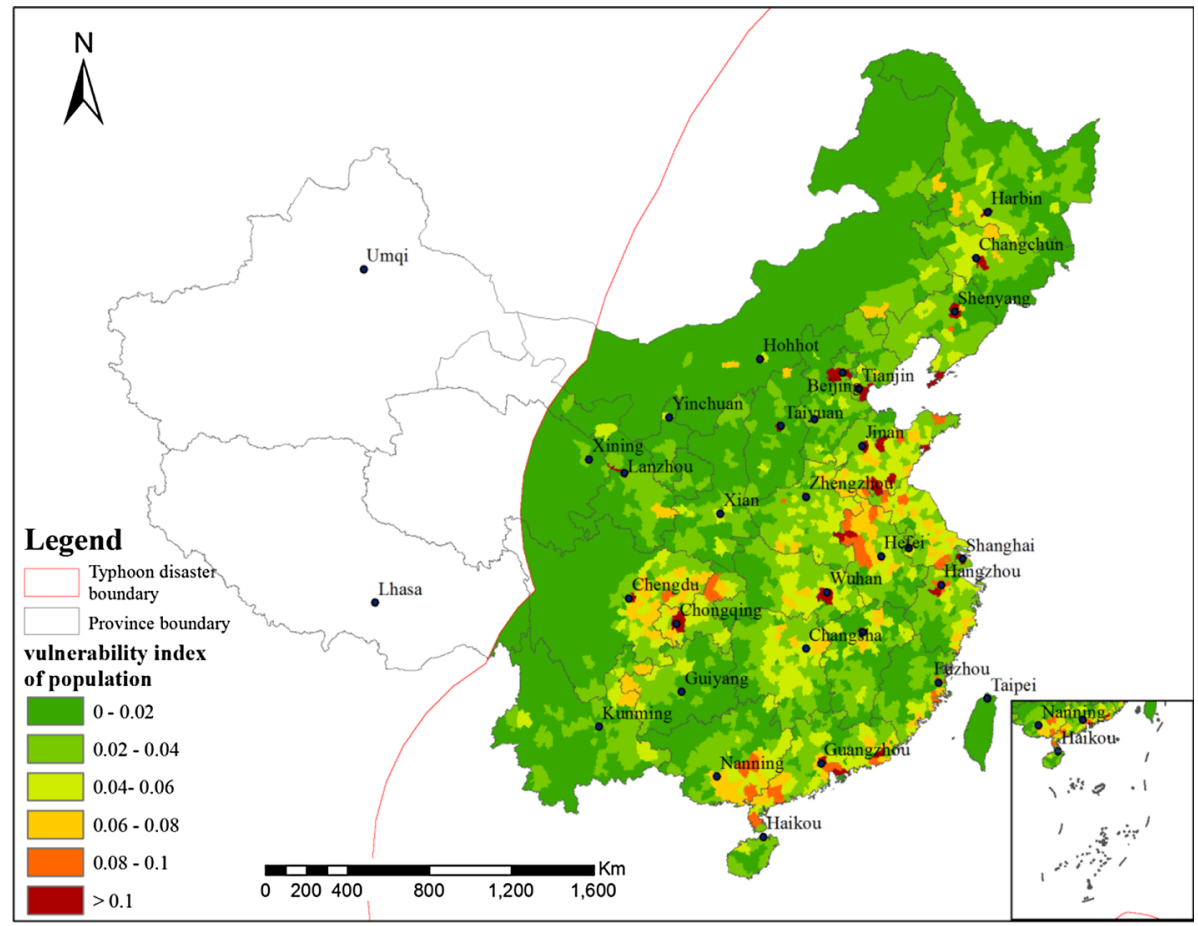

Fig. 3 The distribution of vulnerability indexes of population

Fig. 1 shows the distribution of typhoon hazard index $(T)$ across China, based on the typhoon landfalls that occurred between years 1980 and 2012. The map shows that the highest typhoon hazard index can be found in Taiwan, in which typhoons landed almost 300 times. Among the provinces of mainland China, namely Jiangsu, Shanghai, Zhejiang, Fujian, Guangdong, Hainan and some parts of areas in Anhui, Guangxi, Hunan, Jiangxi provinces have seen their typhoon hazard indexes over 80 . A counter-intuitive finding was that so many inland provinces were also severely affected by typhoons and their typhoon risk indexes range from 1 to 30. Those inland provinces affected by typhoons include Yunnan, Guizhou, Chongqing, Sichuan, Wuhan, Qinghai, Gansu, Ningxia, Shanxi, Henan, Shanaxi, Shandong, Beijing, Tianjin, Hebei, Inner Mongolia, Jilin, Liaoning and Heilongjiang provinces. Two clear patterns were also shown that the typhoon hazard index decreased from east coastal to west inland and from the south to the north of China.

\subsection{Vulnerability of key factors}

\subsubsection{Vulnerability of population}

It is well known that China has the largest population in the world. The population densities across typhoon-affected regions in China were mapped as shown in Fig. 2. The distribution of the population is extremely uneven within China because most of the population reside in the east part of China. This study shows that $97 \%$ of the population 


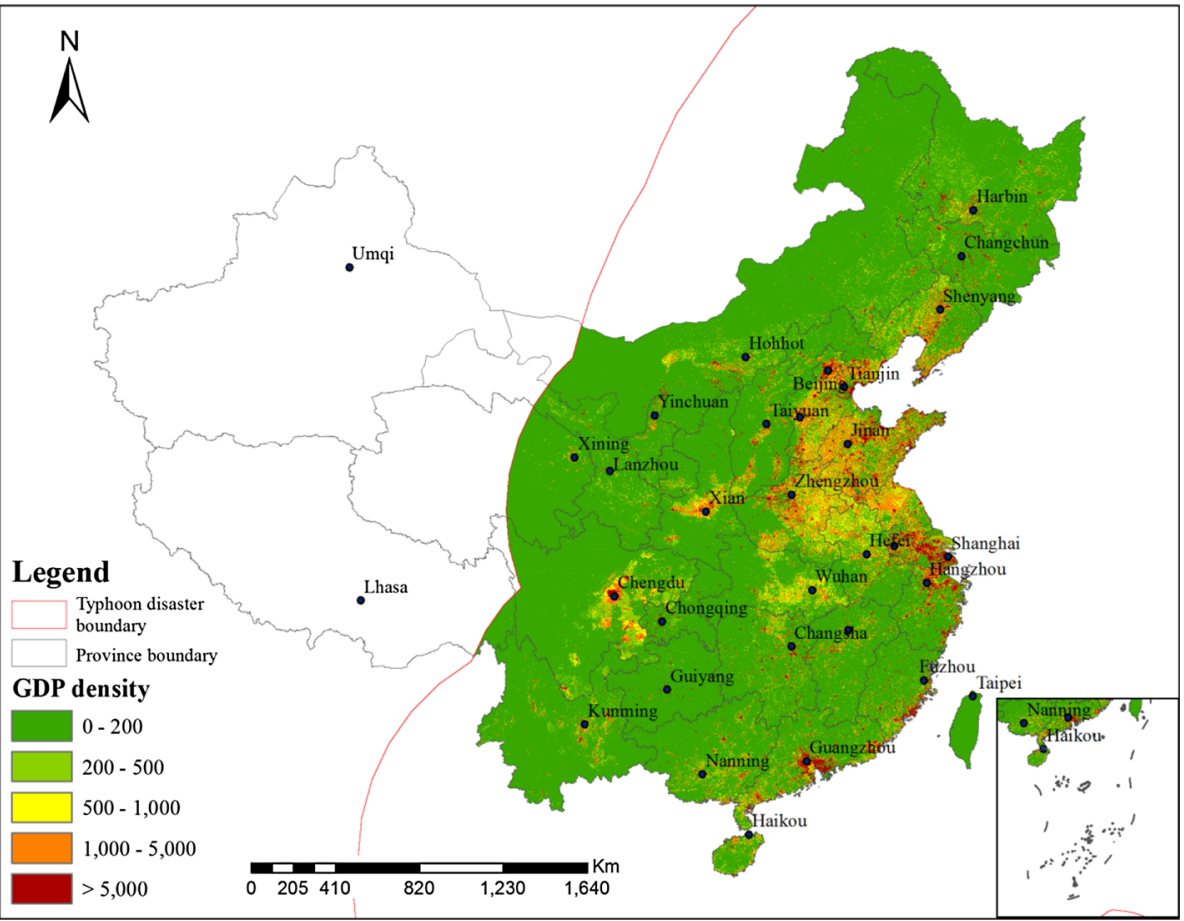

Fig. 4 The distribution of GDP densities in typhoon-affected regions

were affected by typhoons that happened between 1980 and 2012. The population density in the typhoon-affected area was mapped as shown in Fig. 3. For the larger cities that are located in the eastern part of China, their population densities are larger than 700 persons/ $\mathrm{km}^{2}$. Their vulnerability to typhoons is much higher with an index over 0.1 . These large cities include Beijing, Tianjin, Shanghai, Shijiazhuang and Guangzhou, which, in total, accommodate over 235 million people. This represents $18.8 \%$ of the total population of China. There are also several regions that have a higher population density, ranging between 500 and 700 persons $/ \mathrm{km}^{2}$. This can be found in Jing-Jin-Tang region, Huabei Plain, Yangtze Delta, Zhujiang Delta, Sichuan Basin and coastal regions of Zhejiang and Fujian. The vulnerability indexes in these regions range from 0.06 to 0.1 . Total population for these regions is 341 million, which accounts for $27.3 \%$ of the total population of China. There are also many regions whose population density ranges from 401 to 500 persons $/ \mathrm{km}^{2}$ and their vulnerability indexes range between 0.04 and 0.06 . A map of population vulnerability is shown in Fig. 3 .

\subsubsection{Vulnerability of GDP}

Nearly ninety-nine per cent (98.9 \%) of GDP was from typhoon-affected regions between 1980 and 2012. The distribution of GDP densities in typhoon-affected regions is shown in Fig. 4, and the distribution of vulnerability indexes of GDP is shown in Fig. 5. Those super large cities with the highest GDP densities, such as Beijing, Tianjin, Jinan, Xi'an, 


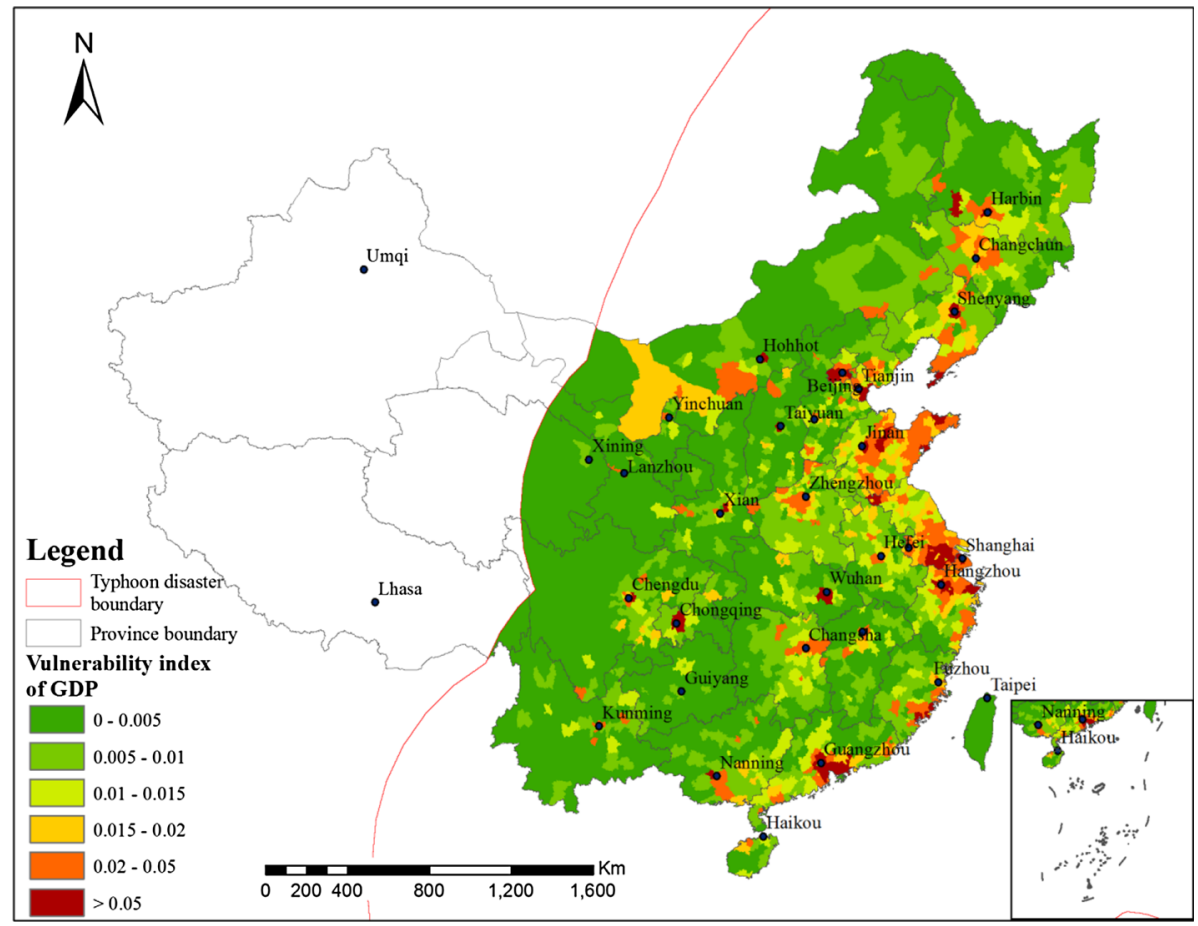

Fig. 5 The distribution of vulnerability indexes of GDP

Shanghai, Hangzhou and Guangzhou have the highest vulnerability indexes, which were over 0.05 . On average, the GDP densities of these above-mentioned super large cities were all above 50 million Chinese Yuan per square kilometre $\left(\mathrm{CNY} / \mathrm{km}^{2}\right)$. Other regions include the southern part of Liaoning province, Jing-Jin-Tang region, Shandong peninsular, Yangtze River Delta, Zhujiang River Delta whose GDP densities ranged from 10 to 50 million $\mathrm{CNY} / \mathrm{km}^{2}$ with vulnerability indexes from 0.02 to 0.05 . Regions include central Liaoning province, southern Hebei province, eastern part of Henan province, western part of Anhui province, eastern part of Hunan province and eastern part of Sichuan province. All their GDP vulnerability indexes were between 0.015 and 0.02 and their GDP ranged from 5 to 10 million $\mathrm{CNY} / \mathrm{km}^{2}$.

\subsubsection{Vulnerability of road network}

The road network has not been evenly developed across China. In general, the roads in eastern China are much better than those of central and western China, which means the density of the highways and the national roads is higher in eastern China compared to those in central and western China (Cheng et al. 2007). Both the landscape and the regional economy development have contributed to these differences. The densities of different types of roads are shown in Fig. 6.

The vulnerability indexes of road systems are shown in Fig. 7. This result reveals that those transportation pivots in China include Shenyang, Beijing, Tianjin, Jinan, Shanghai, Hangzhou, Guangzhou and Chongqing, and their vulnerability levels were very high with 


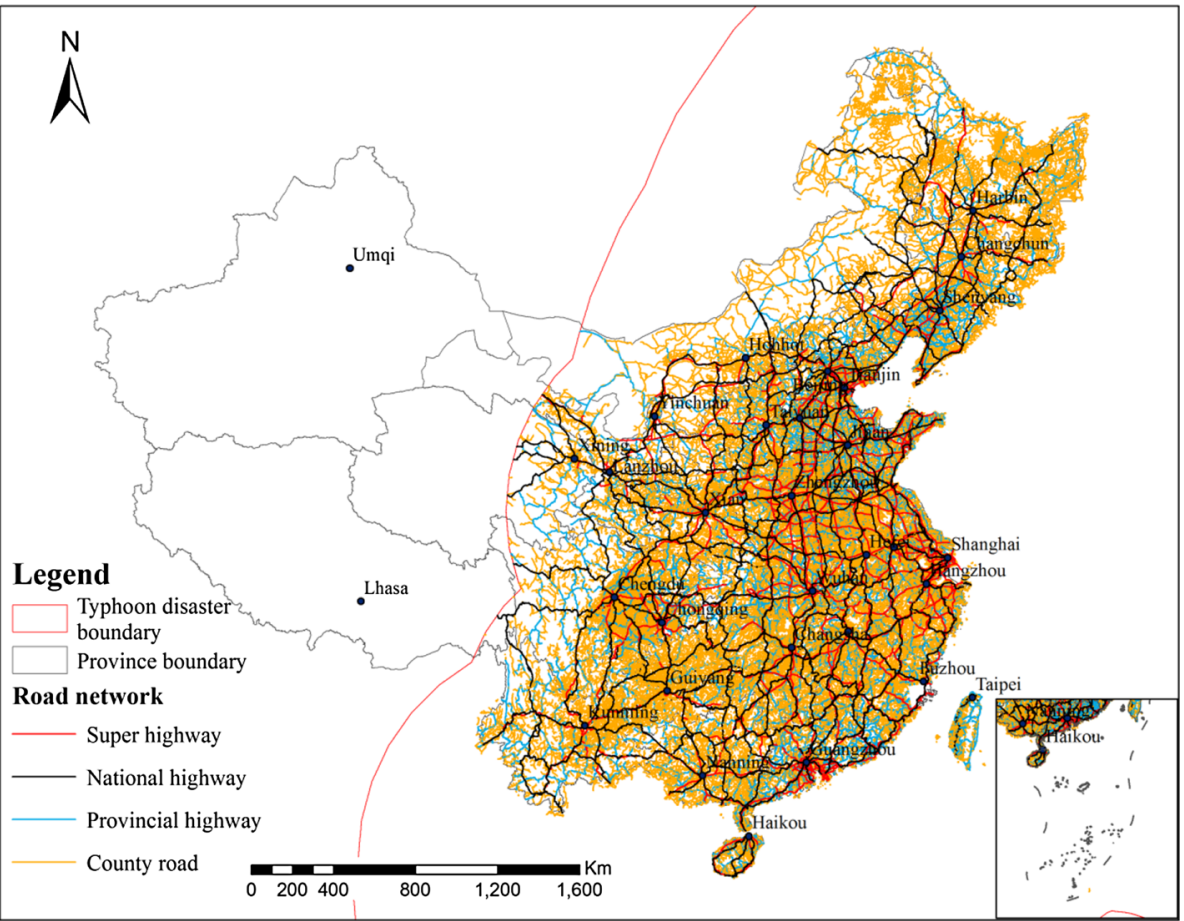

Fig. 6 The distribution of roads in the typhoon-affected regions in China

vulnerability indexes above 0.04 . The regions with well-developed highways, national roads and provincial roads also indicated high vulnerability indexes, with a range between 0.03 and 0.04 . These regions include central and southern Liaoning province, Huabei Plain, Yangtze River delta, Zhujiang river delta and the Sichuan basin.

\subsubsection{Vulnerability of land use}

A map of land-use types for the regions affected by typhoons is presented in Fig. 8. As shown in the map, the built-up areas in the super large cities such as Beijing, Tianjin, Tangshan, Shijiazhuang, Shanghai, Nanjing, Guangzhou and Shenzhen are above $30 \%$. Regions whose built-up areas range between 10 and $30 \%$ are Jing-Jin-Tang, Yangtze River Delta and the Zhujiang River Delta. These regions have been undergoing a fast urbanisation process during the last decade. Most of the croplands in China are located in the Northeast Plain, the Huang-Huai-Hai Plain, the Yangtze River Delta and the Sichuan Basin. These include the areas of south-west of Heilongjiang province, the areas between Jilin and Liaoning provinces, the southern part of Hebei province, the eastern part of Henan province and the western part of Anhui province, with croplands accounting for $80 \%$ of their respective land areas. The vulnerability indexes of land use in the typhoon-affected regions were mapped and are shown in Fig. 9. It shows that the regions with a higher proportion of both built-up areas and cropland areas have the highest vulnerability indexes with a range between 0.025 and 0.03 . These regions include Jing-Jin-Tang, Huang-Huai- 


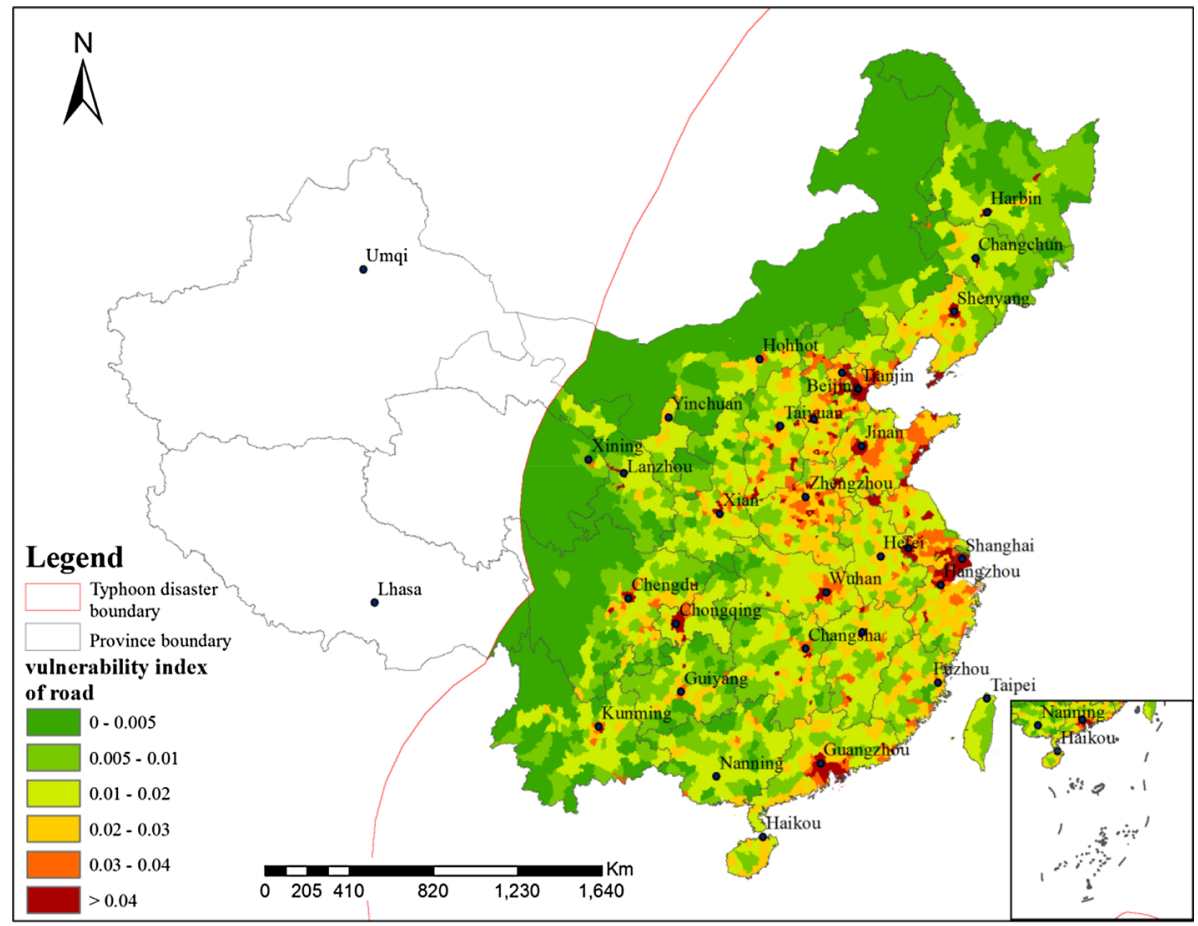

Fig. 7 The map of vulnerability indexes of road network

Hai Plain and Yangtze River Delta. In comparison, the vulnerability indexes of the Northeast Plain, Sichuan basin and Zhujiang River Delta were also high with a range between 0.02 and 0.025 . Their high vulnerability indexes were attributed to a high proportion of cropland in these regions.

\subsubsection{Vulnerability of the entities exposed to typhoons}

The assessment of the vulnerability of the entities exposed to typhoons was achieved by integrating the vulnerabilities of the population, GDP, road network and land use (See Eq. 4). The overall vulnerability index of China to typhoons was mapped as shown in Fig. 10. As it is shown in the map, the super large and large cities in east China all indicate high vulnerability with indexes over 0.12 . These cities are Beijing, Tianjin, Shijiazhuang, Taiyuan, Qingdao, Zhengzhou, Suzhou, Changsha, Xi'an, Shanghai, Nanjing, Hefei, Hangzhou, Zhumadian, Xinxiang, Xingtai, Nanchang, Chongqing, Guangzhou and Nanjing. Their high vulnerabilities were attributed to a high population density, GDP and builtup areas. Among the large cities, namely Harbin, Changchun, Shenyang, Beijing, Tianjin, Jinan, Shanghai, Hangzhou, Suzhou, Chongqing, Guangzhou, vulnerability indexes were over 0.2. This was due to the highest density of population and GDP. The following regions excluding cities have relative higher vulnerability indexes of between 0.08 and 0.12, and they include Huang-Huai-Hai Plain, Yangtze River Delta, Zhujiang River Delta and the Sichuan Basin. 


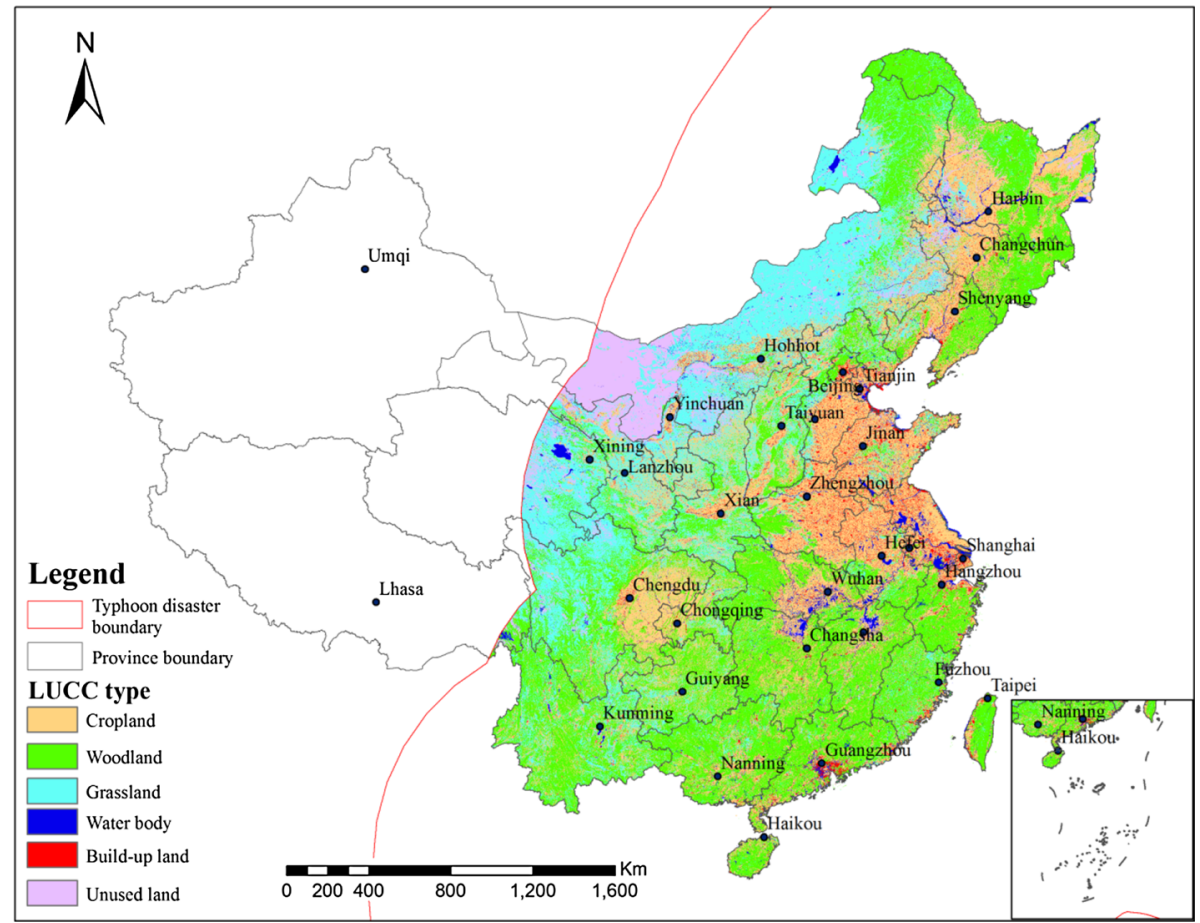

Fig. 8 Land-use types in the typhoon-affected regions in China

\subsection{Typhoon risk}

The typhoon risk indexes were calculated based on Eq. (2) and then were mapped as shown in Fig. 11. The map clearly shows that the typhoon risk in China takes on a decreasing trend from the south-east coastal region to the north-west inland. In addition, the whole of China can be divided into four zones based on regional typhoon risk indexes, which are low-risk area, medium-risk area, high-risk area and very high-risk area. The coastal regions of south-east China have the highest risk indexes, which were above 0.2. Within these areas, some of the largest cities in China such as Shanghai, Hangzhou, Nanjing, Fuzhou, Guangzhou and Haikou have typhoon risk indexes above 0.3. As a result, these provinces, which include Jiangsu, Zhejiang, Fujian, Guangdong and Hainan, are regarded as very high-risk regions affected by typhoons.

The high typhoon risk regions include Shandong peninsular, north-west of Jiangsu province, Anhui province, eastern and central Henan province, south-east of Hubei province, Jiangxi province, south-west of Zhejiang province, central and western part of Fujian province, western part of Guangdong province and south part of Guangxi province. The typhoon risk indexes of the high-risk regions were in a range of 0.1-0.2.

The medium-level typhoon risk regions cover central and western Hunan province, north-western Guangxi province, south-eastern Henan, Hubei, Guizhou and Yunnan provinces. For these regions, the typhoon risk indexes were in the range of $0.05-0.1$. There were also regions only affected by typhoons slightly with typhoon risk indexes $<0.05$. These regions are regarded as low-risk regions, which are shown in the map as a green belt. 


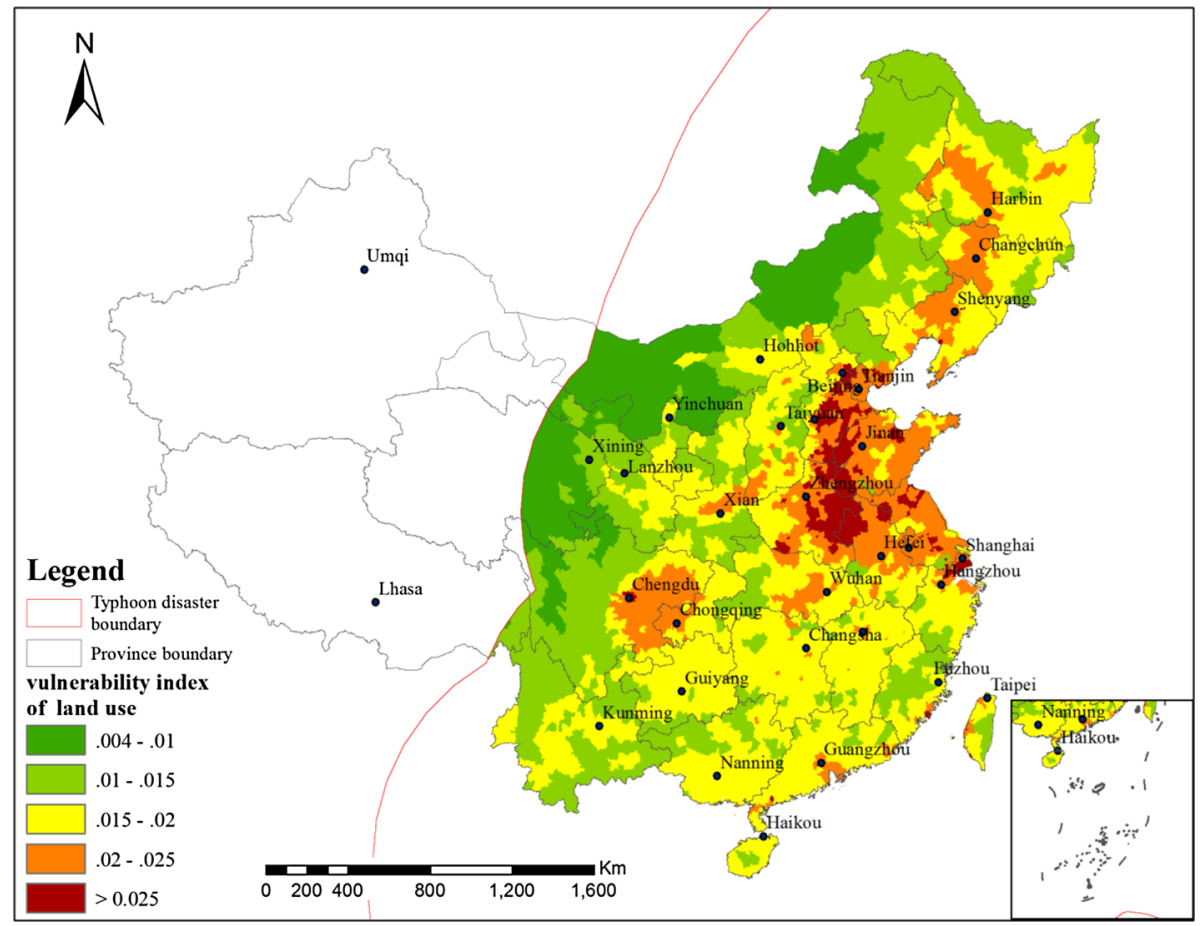

Fig. 9 The map of vulnerability index of land use

\section{Conclusion and discussion}

Typhoons are considered to be extremely devastating natural hazards in China. Most of the prior research has either paid attention to the regions of eastern coastal areas of China or just focussed on a regional area such as one or two of the cities. However, typhoon risk extends far beyond the coastal regions of China, and an overall assessment of typhoon risks at a national level is urgently needed in order to mitigate the risk of typhoon damage. For this reason, this research was conducted to assess the typhoon risk across China. This has been achieved by considering population vulnerabilities, GDP, road network and land use in the typhoon-affected regions based on typhoon data within the timeframe of 1980-2012. The key conclusions generated by this research are that:

1. High typhoon risk areas are concentrated in the south-east coastal regions of China, especially for Taiwan, Hainan, Shanghai, Zhejiang, Fujian, Guangdong and Jiangxi. These areas all experienced over 80 typhoon landfalls during 1980-2012. However, based on our calculations, the typhoon-affected regions were much larger than people generally expected. The area affected by typhoons between 1980 and 2012 was 5.91 million $\mathrm{km}^{2}$, which accounts for $61 \%$ of the total land area of China. This will help to attract the attention of decision-makers for a systemic typhoon risk mitigation plan and associated implementation. For instance, the systemic understanding of typhoon risk across China will enable 


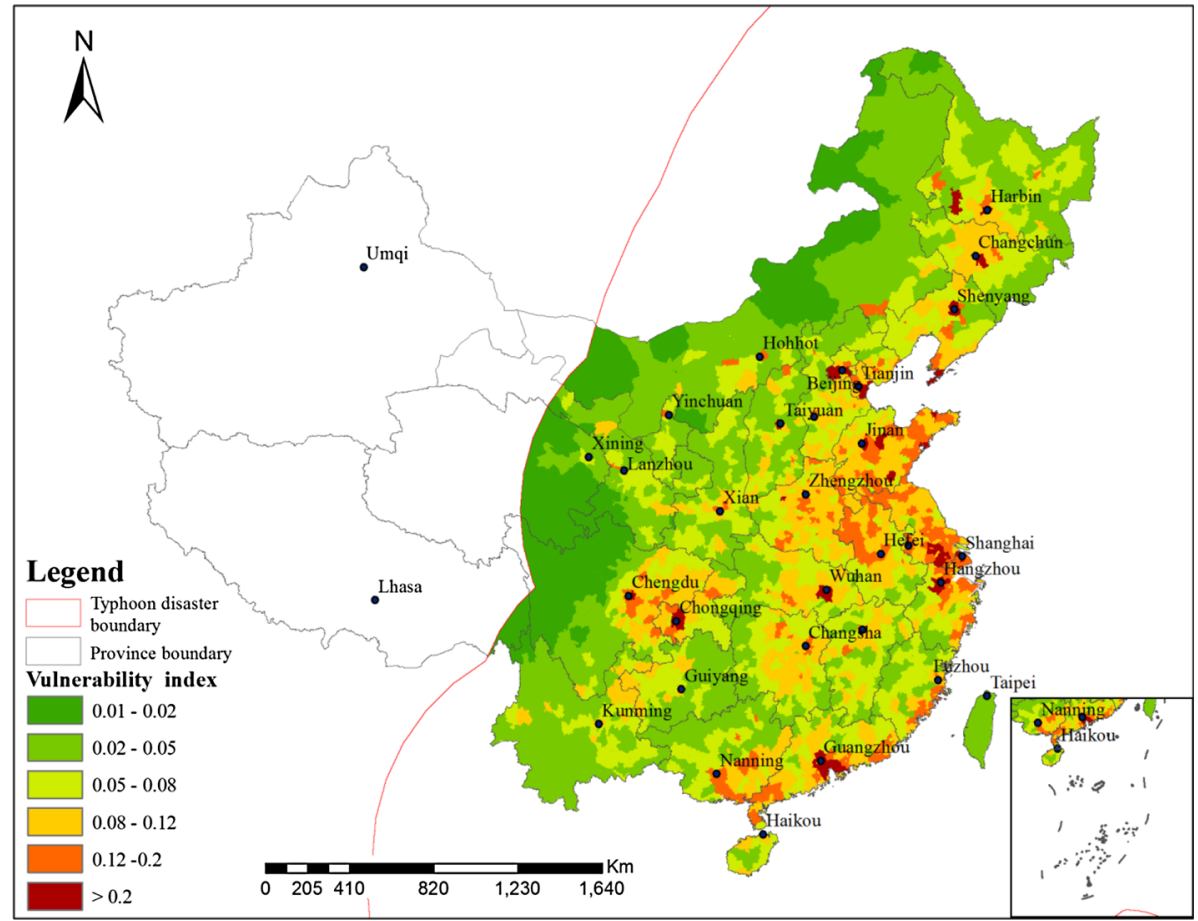

Fig. 10 The map of vulnerability indexes of the entities exposed to typhoons

Central Government to allocate available funding efficiently to where it needs best. It will also enable local Government to make informed decision on the industry planning in order to minimise the scale of industry that is sensitive to typhoon hazards. The assessing typhoon risk at national level will not only improve people's awareness of typhoon hazards, but also facilitate regional collaboration for typhoon hazard prevention.

2. The vulnerability indexes of key factors and the whole entities across typhoonaffected regions were calculated as part of this research. Furthermore, we have also integrated all the information into a map using GIS analysis and mapping techniques. The mapping of vulnerability and risk is a highly encouraged practice by the United Nations. The contribution of this research also rests on that vulnerability indexes of cities and regions in China were calculated in this research. Furthermore, reasons underlying their vulnerabilities to typhoons were also reported in this paper. Cities like Harbin, Changchun, Shenyang, Beijing, Tianjin, Jinan, Shanghai, Hangzhou, Suzhou, Chongqing and Guangzhou have vulnerability indexes above 0.2 , while Huang-Huai-Hai Plain, Yangtze River Delta, Zhujiang River Delta and Sichuan Basin (excluding larger cities) all have relative higher vulnerability indexes of between 0.08 and 0.12 .

3. Regions affected by typhoons were zoned to enable policy decisions to be formulated based on the scales of typhoon risk indexes. Detailed zoned regions are as follows: 


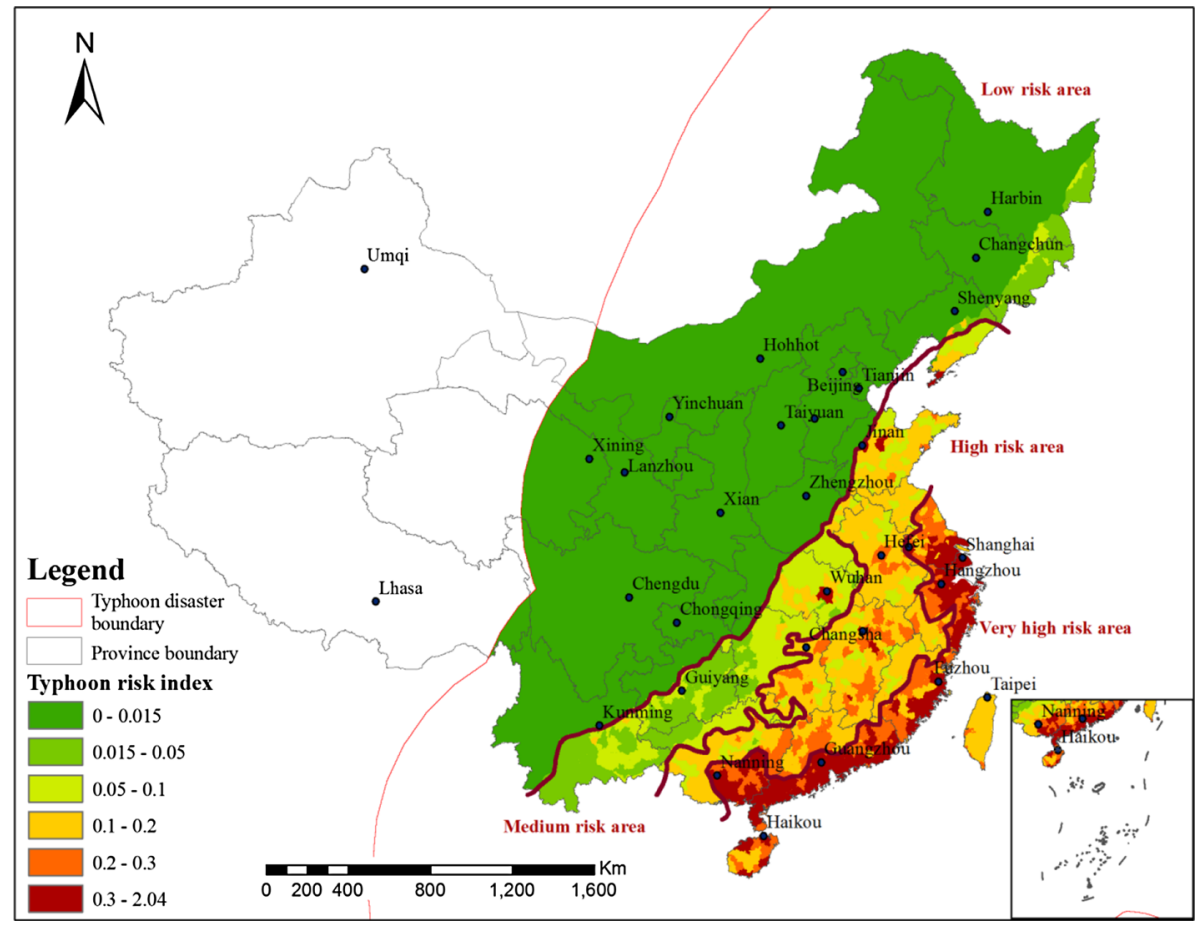

Fig. 11 Map of typhoon risk indexes in China

A: the very high typhoon risk regions are those with a typhoon risk index above 0.2. These areas are mainly located in the Shanghai, Jiangsu, Zhejiang, Fujian, Guangdong and Hainan provinces.

B: regions with a typhoon risk index between 0.1 and 0.2 are regarded as high typhoon risk regions. They include Shandong peninsula, north-west of Jiangsu province, Anhui province, central and east part of Henan province, south-east of Hubei province, Jiangxi province, south-west of Zhejiang province, central and west of Fujian province, west Guangdong province and south-east of Guangxi province.

C: Regions with typhoon risk indexes ranging from 0.05 to 0.1 were defined as medium-typhoon-risk regions. These regions cover central and western Hunan province, north-western Guangxi province, south-eastern Henan, Hubei, Guizhou and Yunnan provinces.

D: low typhoon-risk regions are those typhoon-affected areas to the west of the line drawn between Harbin and Kunming. These regions have typhoon risk indexes $<0.05$.

The authors fully understand the limitations of this research. Due to data limitation on a national scale, the factors considered in this research are mostly at macrolevel. In addition, the exposure factor has to be incorporated into the weights to assess the vulnerability of both the factors and the whole entities. In terms of our future research, we have intentions of including the coping capacity of regions and several meteorological factors in 
calculation of the typhoon-risk index and paying attention to the dynamics of regional vulnerability induced by social and economic development.

Acknowledgments This research was partly supported and funded by three projects, and they were the National Key Project of Scientific and Technical Supporting Programs(No. 2013BAC03B01); the Natural Science Foundation of Chongqing (No. 2010JJ0069); and the Key National Project of High-resolution Earth Observation System (Grant No. 05-Y30B02-9001-13/15-10).

\section{References}

Chen B, Zhong C (2010) A preliminary study on risk loss degree assessment of natural hazards. J Catastrophol 25:1-5

Cheng Q, Hu D, Yi Z (2007) Analysis on spatial difference of land transport density and its change in China. Geogr Geo-Inf Sci 23:71-76

Chia HH, Ropelewski C (2002) The interannual variability in the genesis location of tropical cyclones in the northwest Pacific. J Clim 15:2934-2944

Chu H-J, Liau C-J, Lin C-H, Su B-S (2012) Integration of fuzzy cluster analysis and kernel density estimation for tracking typhoon trajectories in the Taiwan region. Expert Syst Appl 39:9451-9457

Davidson RA, Lambert KB (2001) Comparing the hurricane disaster risk of US coastal counties. Nat Hazards Rev 2:132-142

Dong J, Zhuang D, Xu X, Ying L (2008) Integrated evaluation of urban development suitability based on remote sensing and GIS techniques-a case study in Jingjinji Area, China. Sensors 8:5975-5986

Du X, Lin X (2012) Conceptual model on regional natural disaster risk assessment. Proced Eng 45:96-100

Emanuel K (2003) Tropical cyclones. Annu Rev Earth Planet Sci 31:75-104

Emanuel K (2005) Increasing destructiveness of tropical cyclones over the past 30 years. Nature 436:686-688

Feng L-H, Luo G-Y (2009) Analysis on fuzzy risk of landfall typhoon in Zhejiang province of China. Math Comput Simul 79:3258-3266

Fumin R, Gleason B, Easterling D (2002) Typhoon impacts on China's precipitation during 1957-1996. Adv Atmos Sci 19:943-952

Hashimura R, Takikawa K (2012) Forecasting of damage level of maritime structures caused by typhoon 9918 based on improved CP method. APCBEE Proced 1:272-278

ISDR (2004) Living with risk: a global review of disaster reduction initiative. United Nations Inter-Agency Secretariat of the International Strategy for Disaster Reduction, Geneva

Jones SC et al (2003) The extratropical transition of tropical cyclones: forecast challenges, current understanding, and future directions. Weather Forecast 18:1052-1092

Liu D, Pang L, Xie B (2009) Typhoon disaster in China: prediction, prevention, and mitigation. Nat Hazards 49:421-436

Liu J et al (2010) Spatial patterns and driving forces of land use change in China during the early 21 st century. J Geogr Sci 20:483-494

Liu Y, Chen Z, Wang J, Hu B, Ye M, Xu S (2012) Large-scale natural disaster risk scenario analysis: a case study of Wenzhou City, China. Nat Hazards 60:1287-1298

Liu X, Chen G, Chang Y, Liu K, Zhang L, Xu L (2013) Analyses and countermeasures of deepwater drilling riser grounding accidents under typhoon conditions. Pet Explor Dev 40:791-795

Liu J et al (2014) Spatiotemporal characteristics, patterns, and causes of land-use changes in China since the late 1980s. J Geogr Sci 24:195-210

Matsuura T, Yumoto M, lizuka S (2003) A mechanism of interdecadal variability of tropical cyclone activity over the western North Pacific. Clim Dyn 21:105-117

Meng Z, Chen L, Xu X (2002) Recent progress on tropical cyclone research in China. Adv Atmos Sci 19:103-110

Naughton B (2007) The Chinese economy: transitions and growth. MIT press, Cambridge, Massachusetts, pp 114-135

Ni X, Nan Y (2014) Comprehensive assessment of geological disasters risk in Changbai mountain region based on GIS. J Nat Disasters 23:112-120

Niu H, Liu M, Lu M, Quan R, Zhang L, Wang J (2011) Risk assessment of typhoon disasters in China coastal area during last 20 years. Sci Geogeaphica Sinica 31:764-768 
Peduzzi P, Dao H, Herold C, Mouton F (2009) Assessing global exposure and vulnerability towards natural hazards: the disaster risk index. Nat Hazards Earth Syst Sci 9:1149-1159

Saaty TL (2008) Decision making with the analytic hierarchy process. Int J Serv Sci 1:83-98

State Statistic Bureau (2011) Statistic yearbook, 1st edn. China Statistic Press, Beijing

Tillman CW, Sivillo JK, Frolov SA (2010) Managing typhoon related crop risk at WPC. Agric Agric Sci Proced 1:204-211

Tsai C-H, Chen C-W (2011) The establishment of a rapid natural disaster risk assessment model for the tourism industry. Tour Manag 32:158-171

UNDP/BCPR (2004) Reducing disaster risk: a challenge for development. United Nations Development Programme, Bureau for Crisis Prevention Recovery, New York

Wang B, Xu Y, Bi B (2007) Forecasting and warning of tropical cyclones in China. Data Sci J 6:S723-S737

Wang J, Chen Z, Xu S, Hu B (2013) Medium-scale natural disaster risk scenario analysis: a case study of Pingyang County, Wenzhou, China. Nat Hazards 66:1205-1220

Wu M-C, Yeung K-H, Chang W-L (2006) Trends in western North Pacific tropical cyclone intensity. Eos Trans AGU 87:537-538

Xinhua News (2014a) Typhoon Matmo kills 13 in China (27 July 2014). Xinhua News. http://news. xinhuanet.com/english/china/2014-07/27/c_133513497.html. Accessed 31 July 2014

Xinhua News (2014b) Typhoon Rammasun kills 62 in China (26 July 2014). Xinhua News. http://news. xinhuanet.com/english/china/2014-07/25/c_133510287.html. Accessed 31 July 2014

Xu L, Meng X, Xu X (2014) Natural hazard chain research in China: a review. Nat Hazards 70:1631-1659

Zhang Q, Zhang W, Chen YD, Jiang T (2011) Flood, drought and typhoon disasters during the last halfcentury in the Guangdong province, China. Nat Hazards 57:267-278

Zhang W-Z, Hong H-S, Yan X-H (2013) Typhoons enhancing northward transport through the Taiwan Strait. Cont Shelf Res 56:13-25

Zhang S, Nishijima K, Maruyama T (2014) Reliability-based modeling of typhoon induced wind vulnerability for residential buildings in Japan. J Wind Eng Ind Aerodyn 124:68-81

Zhou W, Yin K, Harrison PJ, Lee JH (2012) The influence of late summer typhoons and high river discharge on water quality in Hong Kong waters. Estuar Coast Shelf Sci 111:35-47 